\title{
Editorial
}

\section{A Change in Scope and a Call for Papers}

One of the important responsibilities of the Editorial Board is to evaluate the scope of papers published in JNeurosci regularly. As neuroscience researchers, we want to ensure the journal reflects the evolution we see in the field and to ensure the journal remains relevant and interesting to our readers, editors, and ourselves.

Over the course of these discussions, the Editorial Board has stressed how important methodological advances are to scientific discovery and decided that it was time to extend the scope of JNeurosci to include papers that provide new ways to study neurobiological problems. We already receive many strong submissions in this area, but historically we have not considered methodological advances for publication in JNeurosci without a novel neurobiological finding. While the journal does publish reviews of some techniques in the TechSights section, we have not considered novel methods on their own for publication.
We are noting this change in scope publicly because authors whose methodological papers have not been sent for external peer review in the past should know this is a formal change in policy that will shift the editorial process going forward. These papers should still be submitted as regular Research Manuscripts and follow the same preparation guidelines.

The Editorial Board has also identified several topics we would like to see more of in the journal. Authors should note in their submission cover letter if their article is in a highlighted topic. These submissions will receive a full, external peer review. Look out for a call for papers with additional details soon.

\section{(iD) Marina Picciotto,}

EiC, JNeurosci

https://doi.org/10.1523/JNEUROSCI.0038-22.2022 